\title{
LINKED DETERMINANTAL LOCI AND LIMIT LINEAR SERIES
}

\author{
JOHN MURRAY AND BRIAN OSSERMAN
}

\begin{abstract}
We study (a generalization of) the notion of linked determinantal loci recently introduced by the second author, showing that as with classical determinantal loci, they are Cohen-Macaulay whenever they have the expected codimension. We apply this to prove Cohen-Macaulayness and flatness for moduli spaces of limit linear series, and to prove a comparison result between the scheme structures of Eisenbud-Harris limit linear series and the spaces of limit linear series recently constructed by the second author. This comparison result is crucial in order to study the geometry of Brill-Noether loci via degenerations.
\end{abstract}

\section{INTRODUCTION}

The theory of limit linear series for curves of compact type was developed by Eisenbud and Harris in a series of papers in the 1980's, with the foundational definitions and results appearing in [EH86]. They were able to give spectacular applications (see for instance [EH87a] and [EH87b]), despite the fact that the moduli space of limit linear series they constructed for families of curves was not proper. However, for finer analyses, it becomes important to have a proper moduli space. This arises for instance when one wants to carry out intersection theory calculations on moduli spaces of linear series, as in Khosla [Kho], or when one wants to study the geometry of moduli spaces of linear series, as in the current work of CastorenaLopez-Teixidor [CLT] and Chan-Lopez-Pflueger-Teixidor [CLPT]. A major step in this direction was accomplished in [Oss14b], when an equivalent definition of limit linear series was introduced, leading to the first proper moduli spaces in families. However, while the new definition was shown to agree with the Eisenbud-Harris definition on a set-theoretic level, the scheme structures are difficult to compare directly. The Eisenbud-Harris scheme structure is more amenable to explicit calculation, but without knowing that the two scheme structures agree, one cannot carry through the arguments of [CLT] and [CLPT], because different non-reduced structures in the special fiber will typically affect more elementary aspects of the generic fiber, such as connectedness or the genus.

In the present paper, we address this issue by showing that under the typical circumstances considered in limit linear series arguments, the two scheme structures do in fact agree. We further show that when they have the expected dimension, limit linear series spaces are Cohen-Macaulay and flat. Our arguments center around an analysis of the "linked determinantal loci" introduced in Appendix A of [Oss14a] in order to prove smoothing theorems for limit linear series. In fact, we study a more general definition than the one considered in [Oss14a], which is also a generalization of classical determinantal loci. A preliminary definition is the following.

The second author was partially supported by Simons Foundation grant \#279151 during the preparation of this work. 
Definition 1.1. Let $S$ be a scheme, and $d, n$ be positive integers. Suppose that $\mathscr{E}_{1}, \ldots, \mathscr{E}_{n}$ are vector bundles of rank $d$ on $S$ and we have morphisms

$$
f_{i}: \mathscr{E}_{i} \rightarrow \mathscr{E}_{i+1}, \quad f^{i}: \mathscr{E}_{i+1} \rightarrow \mathscr{E}_{i}
$$

for each $i=1, \ldots, n-1$. Given $s \in \Gamma\left(S, \mathscr{O}_{S}\right)$, we say that $\mathscr{E}_{\bullet}=\left(\mathscr{E}_{i}, f_{i}, f^{i}\right)_{i}$ is an $s$-linked chain if the following conditions are satisfied:

(I) For each $i=1, \ldots, n$,

$$
f_{i} \circ f^{i}=s \cdot \mathrm{id}, \text { and } f^{i} \circ f_{i}=s \cdot \mathrm{id} .
$$

(II) On the fibers of the $\mathscr{E}_{i}$ at any point with $s=0$, we have that for each $i=1, \ldots, n-1$,

$$
\operatorname{ker} f^{i}=\operatorname{im} f_{i}, \text { and } \operatorname{ker} f_{i}=\operatorname{im} f^{i} .
$$

(III) On the fibers of the $\mathscr{E}_{i}$ at any point with $s=0$, we have that for each $i=1, \ldots, n-2$,

$$
\operatorname{im} f_{i} \cap \operatorname{ker} f_{i+1}=(0), \text { and } \operatorname{im} f^{i+1} \cap \operatorname{ker} f^{i}=(0) .
$$

We then define linked determinantal loci as follows.

Definition 1.2. Let $\mathscr{E}_{\bullet}$ be an $s$-linked chain on a scheme $S$. Given $r, r_{1}, r_{n}>0$, suppose $\mathscr{F}_{1}, \mathscr{F}_{n}$ are vector bundles of rank $r_{1}$ and $r_{n}$ respectively, and let $g_{1}: \mathscr{E}_{1} \rightarrow \mathscr{F}_{n}$ and $g_{n}: \mathscr{E}_{n} \rightarrow \mathscr{F}_{n}$ be any morphisms. Then the associated linked determinantal locus is the closed subscheme of $S$ on which the induced morphisms

$$
\mathscr{E}_{i} \rightarrow \mathscr{F}_{1} \oplus \mathscr{F}_{n}
$$

have rank less than or equal to $r$ for all $i=1, \ldots, n$.

Note that the case $n=1$ and $\mathscr{F}_{n}=0$ recovers the usual notion of determinantal locus. It appears a priori that the codimension could be much larger than for a single determinantal locus, but it turns out that the different determinantal conditions we are imposing are highly dependent. The case considered in Appendix A of [Oss14a] amounted to requiring that $g_{1}$ and $g_{n}$ be quotient maps onto bundles of rank $r$. In that situation, it was shown that the classical codimension bound also applies to linked determinantal loci. However, Cohen-Macaulayness does not appear to be addressable by the methods used in loc. cit. Our main theorem is thus the following:

Theorem 1.3. If $S$ is Noetherian, then in the situation of Definition 1.2, every irreducible component of the linked determinantal locus has codimension at most $(d-r)\left(r_{1}+r_{n}-r\right)$. Moreover, if equality holds, and $S$ is Cohen-Macaulay then the linked determinantal locus is also Cohen-Macaulay.

The proof proceeds by considering a suitable universal version of the linked determinantal locus, and showing in essence that it is a "partial initial degeneration" of the classical universal determinant locus. That is to say, in terms of ideals of minors we show that our locus is defined by zeroing out monomials in each minor according to a certain pattern, and that the resulting initial ideal is the same as in the classical case. In comparison with other previously studied variants of determinantal ideals, this appears to yield a rather distinct direction of generalization.

As mentioned previously, using the construction given in [Oss14a], we conclude from Theorem 1.3 that spaces of limit linear series are flat and Cohen-Macaulay; see 
Theorem 3.1 below. We then conclude in Corollary 3.3 that the two scheme structures on spaces of limit linear series agree under typical circumstances (specifically, when the space has the expected dimension, the open subset of refined limit linear series is dense, and the Eisenbud-Harris scheme structure is reduced). Finally, for the arguments of [CLT] and [CLPT], the crucial consequence is Corollary 3.4. This says that under the same conditions as Corollary 3.3, given a one-parameter smoothing of a curve $X_{0}$ of compact type, with generic fiber $X_{\eta}$, we have a flat, proper moduli space whose special fiber is the Eisenbud-Harris moduli space of limit linear series on $X_{0}$, and whose generic fiber is the usual linear series moduli space on $X_{\eta}$.

\section{LINKED DETERMINANTAL LOCI}

We consider the following situation:

Situation 2.1. Let $R$ be a ring, and $s \in R$. Given $d, n, r_{1}, r_{n}$, and nondecreasing integers $c_{1} \leqslant c_{2} \leqslant \ldots c_{n-1}$, let $R^{\prime}$ be the polynomial ring over $R$ in variables $x_{i, j}$ with $1 \leqslant i \leqslant r_{1}+r_{n}$ and $1 \leqslant j \leqslant d$.

Definition 2.2. For $\ell=1, \ldots, n$, let $A_{\ell}$ be the $\left(r_{1}+r_{n}\right) \times d$ matrix over $R^{\prime}$ defined by

where

$$
\left(A_{\ell}\right)_{i, j}= \begin{cases}s^{e_{1, j, \ell}} x_{i, j}: & i \leqslant r_{1} \\ s^{e_{1, j, \ell}} x_{i, j}: & i>r_{1}\end{cases}
$$

$$
e_{1, j, \ell}=\#\left\{m<\ell: j>d-c_{m}\right\}, \quad \text { and } \quad e_{2, j, \ell}=\#\left\{m \geqslant \ell: j \leqslant d-c_{m}\right\} .
$$

Given $r>0$, let $I_{r}$ be the ideal of $R^{\prime}$ defined by all $(r+1) \times(r+1)$ minors of all the $A_{\ell}$. In addition, let $J_{r}$ be the universal determinantal ideal obtained from the $(r+1) \times(r+1)$ minors of the matrix with $i, j$ entry equal to $x_{i, j}$.

Example 2.3. Consider the case $d=5, n=4, c_{1}=1, c_{2}=3, c_{3}=3, r=2$, $r_{1}=1, r_{n}=2$, and $s=0$. Then the matrices $A_{\ell}$ are as follows:

$$
\begin{array}{rlrll}
A_{1} & =\left[\begin{array}{ccccc}
x_{1,1} & x_{1,2} & x_{1,3} & x_{1,4} & x_{1,5} \\
0 & 0 & 0 & 0 & x_{2,5} \\
0 & 0 & 0 & 0 & x_{2,5}
\end{array}\right], & A_{2}=\left[\begin{array}{ccccc}
x_{1,1} & x_{1,2} & x_{1,3} & x_{1,4} & 0 \\
0 & 0 & x_{2,3} & x_{2,4} & x_{2,5} \\
0 & 0 & x_{3,3} & x_{3,4} & x_{3,5}
\end{array}\right], \\
A_{3}=\left[\begin{array}{ccccc}
x_{1,1} & x_{1,2} & 0 & 0 & 0 \\
0 & 0 & x_{2,3} & x_{2,4} & x_{2,5} \\
0 & 0 & x_{3,3} & x_{3,4} & x_{3,5}
\end{array}\right], & A_{4}=\left[\begin{array}{ccccc}
x_{1,1} & x_{1,2} & 0 & 0 & 0 \\
x_{2,1} & x_{2,2} & x_{2,3} & x_{2,4} & x_{2,5} \\
x_{3,1} & x_{3,2} & x_{3,3} & x_{3,4} & x_{3,5}
\end{array}\right] .
\end{array}
$$

We do not list every minor from each of the $A_{\ell}$, but rather examine a selection of them with representative behavior. The minors from the first three columns of the $A_{\ell}$ are

$$
0, \quad 0, \quad 0, \text { and } x_{1,1} x_{2,2} x_{3,3}-x_{1,1} x_{3,2} x_{2,3}-x_{2,1} x_{1,2} x_{3,3}+x_{3,1} x_{1,2} x_{2,3},
$$

respectively. The minors from the second, third and fourth columns of the $A_{\ell}$ are

$$
\begin{array}{r}
0, \quad x_{1,2} x_{2,3} x_{3,4}-x_{1,2} x_{3,3} x_{2,4}, \quad x_{1,2} x_{2,3} x_{3,4}-x_{1,2} x_{3,3} x_{2,4}, \\
\text { and } x_{1,2} x_{2,3} x_{3,4}-x_{1,2} x_{3,3} x_{2,4},
\end{array}
$$

respectively. Finally, the minors from the last three columns of the $A_{\ell}$ are

$$
0, \quad x_{1,3} x_{2,4} x_{3,5}-x_{1,3} x_{3,4} x_{2,5}-x_{2,3} x_{1,4} x_{3,5}+x_{3,3} x_{1,4} x_{2,5}, \quad 0, \text { and } 0,
$$


respectively.

The key aspects of these minors are the following:

- For each fixed minor position, the corresponding minors of the different $A_{\ell}$ are always equal to zero or a unique nonzero value.

- The unique nonzero value always occurs for some $\ell$, and always contains the "main diagonal" term of the usual universal minor.

- There need not be a unique choice of $\ell$ achieving the unique nonzero minor value, and no single value of $\ell$ generates nonzero minors in all positions.

Note that the uniqueness of the nonzero minor value occurs in spite of the fact that a given nonzero minor may be generated by different patterns of zeroing out entries (as occurs with $A_{2}, A_{3}$ and $A_{4}$ for the minor from the second, third and fourth columns).

Throughout this section, we will use the lexicographic monomial order, induced by the ordering that $x_{i, j}$ comes before $x_{i^{\prime}, j^{\prime}}$ if $i<i^{\prime}$ or $i=i^{\prime}$ and $j<j^{\prime}$. In order to prove Theorem 1.3, the main result is then the following:

Theorem 2.4. In the case $R=k$ is a field, then the initial ideal of $I_{r}$ coincides with the initial ideal of $J_{r}$, and in particular the Hilbert functions coincide. If $s \neq 0$, then also $R^{\prime} / I_{r} \cong R^{\prime} / J_{r}$.

We prove this by exhibiting $I_{r}$ as a flat degeneration of $J_{r}$, using the following construction.

Definition 2.5. Suppose $R=k[t]$, and let $B$ be the $\left(r_{1}+r_{n}\right) \times d$ matrix over $R^{\prime}$ defined by

$$
(B)_{i, j}= \begin{cases}t^{\epsilon_{1, j}} x_{i, j}: & i \leqslant r_{1} \\ t^{\epsilon_{2, j}} x_{i, j}: & i>r_{1}\end{cases}
$$

where

$$
\epsilon_{1, j}=\#\left\{m: j>d-c_{m}\right\}, \quad \text { and } \quad \epsilon_{2, j}=\#\left\{m: j \leqslant d-c_{m}\right\} .
$$

Thus, $\epsilon_{1, j}=e_{1, j, n}$ and $\epsilon_{2, j}=e_{2, j, 1}$.

Example 2.6. In the context of Example 2.3, we have

$$
B=\left[\begin{array}{ccccc}
x_{1,1} & x_{1,2} & t^{2} x_{1,3} & t^{2} x_{1,4} & t^{3} x_{1,5} \\
t^{3} x_{2,1} & t^{3} x_{2,2} & t x_{2,3} & t x_{2,4} & x_{2,5} \\
t^{3} x_{3,1} & t^{3} x_{3,2} & t x_{3,3} & t x_{3,4} & x_{3,5}
\end{array}\right] .
$$

If we consider the minor from the first three columns of $B$, we get

$$
\begin{aligned}
t^{4} x_{1,1} x_{2,2} x_{3,3}-t^{4} x_{1,1} x_{3,2} x_{2,3} & -t^{4} x_{2,1} x_{1,2} x_{3,3} \\
& +t^{4} x_{3,1} x_{1,2} x_{2,3}+t^{8} x_{2,1} x_{3,2} x_{1,3}-t^{8} x_{3,1} x_{2,2} x_{1,3} .
\end{aligned}
$$

Observe that if we factor out $t^{4}$ from this and then set $t=0$ we obtain the nonzero minor from $A_{4}$ of Example 2.3.

The minor from the second, third and fourth columns of $B$ is

$$
\begin{aligned}
t^{2} x_{1,2} x_{2,3} x_{3,4}-t^{2} x_{1,2} x_{3,3} x_{2,4} & -t^{6} x_{2,2} x_{1,3} x_{3,4} \\
& +t^{6} x_{3,2} x_{1,3} x_{2,4}+t^{6} x_{2,2} x_{3,3} x_{1,4}-t^{6} x_{3,2} x_{2,3} x_{1,4},
\end{aligned}
$$

and again factoring out $t^{2}$ and setting $t=0$ recovers the corresponding nonzero minors from $A_{2}, A_{3}$ and $A_{4}$. 
Finally, the minor from the last three columns of $B$ is

$$
\begin{aligned}
t^{3} x_{1,3} x_{2,4} x_{3,5}-t^{3} x_{1,3} x_{3,4} x_{2,5} & -t^{3} x_{2,3} x_{1,4} x_{3,5} \\
& +t^{3} x_{3,3} x_{1,4} x_{2,5}+t^{5} x_{3,3} x_{2,4} x_{1,5}-t^{5} x_{2,3} x_{3,4} x_{1,5},
\end{aligned}
$$

and factoring out $t^{3}$ and setting $t=0$ yields the corresponding nonzero minor from $A_{2}$.

The above, together with similar analysis of the remaining minors, says that in this example the ideal $I_{r}$ is governed in a suitable sense by the single matrix $B$, and it follows that $I_{r}$ is a flat degeneration of the universal determinantal ideal $J_{r}$.

The following key lemma shows that the behavior of Examples 2.3 and 2.6 generalizes.

Lemma 2.7. In the case that $R=k$ is a field and $s=0$, then if we fix $i_{\bullet}=$ $\left(i_{0}, \ldots, i_{r}\right)$ with $1 \leqslant i_{0}<i_{1}<\cdots<i_{r} \leqslant r_{1}+r_{n}$ and $j_{\bullet}=\left(j_{0}, \ldots, j_{r}\right)$ with $1 \leqslant j_{0}<j_{1}<\cdots<j_{r} \leqslant d$, let $g_{i_{\bullet}, j_{\bullet}}$ be obtained from the $\left(i_{\bullet}, j_{\bullet}\right)$ minor of $B$ by factoring out the largest possible power of $t$, and then setting $t=0$.

Then for all $\ell$, the $\left(i_{\bullet}, j_{\bullet}\right)$ minor of $A_{\ell}$ is either equal to 0 or to $g_{i_{\bullet}, j_{\bullet}}$, and the latter occurs at least once. In addition, $g_{i_{\bullet}, j_{\bullet}}$ contains the monomial $x_{i_{0}, j_{0}} x_{i_{1}, j_{1}} \cdots x_{i_{r}, j_{r}}$.

Proof. For each $\ell$, let $A_{\ell}^{\prime}$ be the $(r+1) \times(r+1)$ matrix obtained from $A_{\ell}$ by taking the $(i, j)$ entries with $i \in\left\{i_{0}, \ldots, i_{r}\right\}$ and $j \in\left\{j_{0}, \ldots, j_{r}\right\}$, so that the minor we are considering is $\operatorname{det} A_{\ell}^{\prime}$. Let $m_{1}$ be the number of $w$ such that $i_{w} \leqslant r_{1}$, and $m_{2}$ the number of $w$ such that $i_{w}>r_{1}$, so that $m_{1}+m_{2}=r+1$. Then for any $\ell$, there are $p_{1, \ell}, p_{2, \ell} \geqslant 0$ so that the first $m_{1}$ rows of the matrix $A_{\ell}^{\prime}$ end in $p_{1, \ell}$ zeroes, while the last $m_{2}$ rows begin with $p_{2, \ell}$ zeroes (reading left to right). Explicitly, we have

$$
p_{1, \ell}=\#\left\{w: j_{w}>d-c_{\ell-1}\right\}, \quad p_{2, \ell}=\#\left\{w: j_{w} \leqslant d-c_{\ell}\right\},
$$

where here we should use the convention that $c_{0}=0$ and $c_{n}=d$. In particular, we have $p_{1, \ell+1}+p_{2, \ell}=r+1$ for $\ell=1, \ldots, n-1$. Now, clearly $\operatorname{det} A_{\ell}^{\prime}$ can only be nonzero if $r+1-p_{1, \ell} \geqslant m_{1}$ and $r+1-p_{2, \ell} \geqslant m_{2}$, and in fact, since the individual terms appearing in the determinant are distinct monomials, there cannot be any cancellation, so the converse holds as well. Then $p_{1,1}=0$, so if we choose $\ell$ maximal with $r+1-p_{1, \ell} \geqslant m_{1}$, and if $\ell<n$, then we have $p_{2, \ell}=r+1-p_{1, \ell+1}<m_{1}=$ $r+1-m_{2}$, so in this case $\operatorname{det} A_{\ell}^{\prime} \neq 0$. On the other hand, if $r+1-p_{1, n} \geqslant m_{1}$, then $p_{2, n}=0 \leqslant r+1-m_{2}$, so again $\operatorname{det} A_{n}^{\prime} \neq 0$.

Thus, it remains to show that for any $\ell$ such that $r+1-p_{1, \ell} \geqslant m_{1}$ and $r+1-p_{2, \ell} \geqslant m_{2}$, we have that $\operatorname{det} A_{\ell}^{\prime}=g_{i_{\bullet}, j_{\bullet}}$, and that $g_{i_{\bullet}, j_{\bullet}}$ contains the monomial $x_{i_{0}, j_{0}} x_{i_{1}, j_{1}} \cdots x_{i_{r}, j_{r}}$. Now, both det $A_{\ell}^{\prime}$ and $g_{i_{\bullet}, j_{\bullet}}$ are obtained by omitting some monomials from the determinant of the $(r+1) \times(r+1)$ matrix with entries given by $x_{i_{w}, j_{v}}$, so we can prove the desired statements by explicitly identifying which monomials are included in each. First, for $g_{i_{\bullet}, j}$. we observe that since in $B$ the powers of $t$ in the first $r_{1}$ rows are nondecreasing from left to right, and in the last $r_{n}$ rows are nonincreasing from left to right, it is clear that (within the rows and columns determined by $i_{\bullet}, j_{\bullet}$ ) if we take the top $m_{1}$ entries from the first $m_{1}$ columns (and consequently the remaining $m_{2}$ entries from the last $m_{2}$ columns) we will simultaneously minimize the power of $t$ coming from the top $m_{1}$ rows and the bottom $m_{2}$ rows. In particular, the "diagonal" term obtained from $\left(i_{0}, j_{0}\right),\left(i_{1}, j_{1}\right), \ldots,\left(i_{r}, j_{r}\right)$ achieves the minimal possible power of $t$ in the relevant minor, so $g_{i_{\bullet}, j \bullet}$ contains $x_{i_{0}, j_{0}} x_{i_{1}, j_{1}} \cdots x_{i_{r}, j_{r}}$, as claimed. Moreover, we see that in order for a general term 
coming from $\left(i_{0}, j_{\sigma(0)}\right), \ldots,\left(i_{r}, j_{\sigma(r)}\right)$ for $\sigma \in \operatorname{Sym}(\{0, \ldots, r\})$ to have the minimal power of $t$, we must have $\epsilon_{1, j_{\sigma(0)}}, \ldots, \epsilon_{1, j_{\sigma\left(m_{1}-1\right)}}$ equal (as an unordered set with repetitions) to $\epsilon_{1, j_{0}}, \ldots, \epsilon_{1, j_{m_{1}-1}}$, and similarly for $\epsilon_{2, j_{\sigma\left(m_{1}\right)}}, \ldots, \epsilon_{2, j_{\sigma(r)}}$ and $\epsilon_{2, j_{m_{1}}}, \ldots, \epsilon_{2, j_{r}}$.

Now, suppose that $\epsilon_{1, j_{\sigma(0)}}, \ldots, \epsilon_{1, j_{\sigma\left(m_{1}-1\right)}}$ is not equal to $\epsilon_{1, j_{0}}, \ldots, \epsilon_{1, j_{m_{1}-1}}$. Then let $w \leqslant m_{1}-1$ be minimal such that the entry in column $j_{w}$ is taken from one of the bottom $m_{2}$ rows, so that $\epsilon_{1, j_{w}}$ occurs strictly fewer times in the sequence $\epsilon_{1, j_{\sigma(0)}}, \ldots, \epsilon_{1, j_{\sigma\left(m_{1}-1\right)}}$ than in $\epsilon_{1, j_{0}}, \ldots, \epsilon_{1, j_{m_{1}-1}}$. If $\epsilon_{1, j_{w}}=\epsilon_{1, j_{m_{1}-1}}$, we conclude from minimality of $\epsilon_{1, j_{0}}, \ldots, \epsilon_{1, j_{m_{1}-1}}$ that for some $w^{\prime} \leqslant m_{1}-1$, we have

$$
\epsilon_{1, j_{\sigma\left(w^{\prime}\right)}}>\epsilon_{1, j_{m_{1}-1}}
$$

On the other hand, if $\epsilon_{1, j_{w}}<\epsilon_{1, j_{m_{1}-1}}$, we have that

$$
\epsilon_{2, j_{w}}>\epsilon_{2, j_{m_{1}-1}} \geqslant \epsilon_{2, j_{m_{1}}}
$$

and because we have taken the entry in the $j_{w}$ column from the bottom $m_{2}$ rows, we conclude that for some $w^{\prime} \geqslant m_{1}$, we have

$$
\epsilon_{2, j_{\sigma\left(w^{\prime}\right)}}=\epsilon_{2, j_{w}}>\epsilon_{2, j_{m_{1}}} .
$$

To summarize, we obtain a non-minimal power of $t$ for a given term if and only if $\epsilon_{1, j_{\sigma(w)}} \leqslant \epsilon_{1, j_{m_{1}-1}}$ for all $w<m_{1}$, and $\epsilon_{2, j_{\sigma(w)}} \leqslant \epsilon_{2, j_{m_{1}}}$ for all $w \geqslant m_{1}$. Thus, if we set $a$ so that $d-c_{a}<j_{m_{1}} \leqslant d-c_{a-1}$, and $b$ so that $d-c_{b}<j_{m_{1}+1} \leqslant d-c_{b-1}$, then we are saying simply that a given term appears in $g_{i_{\bullet}, j \bullet}$ if and only if

$$
j_{\sigma(w)} \leqslant d-c_{a-1} \text { for } w<m_{1} \text {, and } j_{\sigma(w)}>d-c_{b} \text { for } w \geqslant m_{1} .
$$

Now we consider the monomials in $\operatorname{det} A_{\ell}^{\prime}$ for $\ell$ such that $r+1-p_{1, \ell} \geqslant m_{1}$ and $r+$ $1-p_{2, \ell} \geqslant m_{2}$. It is clear that a given monomial from entries $\left(i_{0}, j_{\sigma(0)}\right), \ldots,\left(i_{r}, j_{\sigma(r)}\right)$ occurs in $\operatorname{det} A_{\ell}^{\prime}$ if and only if

$$
j_{\sigma(w)} \leqslant d-c_{\ell-1} \text { for } w<m_{1} \text {, and } \quad j_{\sigma(w)}>d-c_{\ell} \text { for } w \geqslant m_{1} .
$$

To compare (2.1) to $(2.2)$, we note that $r+1-p_{1, \ell} \geqslant m_{1}$ is equivalent to saying that $j_{m_{1}} \leqslant d-c_{\ell-1}$, and $r+1-p_{2, \ell} \geqslant m_{2}$ is the same as $p_{2, \ell} \leqslant m_{1}$, which is the same as $j_{m_{1}+1}>d-c_{\ell}$, so we can conclude that $\ell-1 \leqslant a-1$ and $\ell \geqslant b$, which is to say that the relevant range for $\ell$ is $b \leqslant \ell \leqslant a$. It then follows immediately that (2.1) implies (2.2), and we wish to verify the converse.

If $a=b$, the converse is likewise immediate. However, we see from the definitions that if $a>b$, then $d-c_{b}=d-c_{a-1}>d-c_{a}$, and then the first part of (2.1) is equivalent to the second part. In addition, we must have either $\ell=a$ or $c_{\ell}=c_{b}$. In the first case, we have that $c_{a-1}=c_{\ell-1}$, so the first part of (2.2) implies the first part of (2.1), which then implies the second part of (2.1) as well. But in the second case, the second part of (2.2) implies the second part of (2.1), which then implies the first part as well.

Proof of Theorem 2.4. First suppose that $s \neq 0$. We see that in this case, for $\ell<n$ the matrix $A_{\ell+1}$ may be obtained from $A_{\ell}$ by multiplying the righthand $c_{\ell}$ columns by $s$, and dividing the bottom $r_{n}$ rows by $s$. Thus, any given minor of $A_{\ell}$ is a power of $s$ times the corresponding minor of $A_{\ell+1}$, and we concude that $I_{r}$ is simply equal to the ideal generated by the $(r+1) \times(r+1)$ minors of $A_{1}$. As this is obtained from $J_{r}$ by rescaling the variables by powers of $s$, we find that $R^{\prime} / I_{r} \cong R^{\prime} / J_{r}$, and also that the initial ideals and Hilbert functions coincide. 
Now, suppose that $s=0$. According to Lemma 2.7, in this case $I_{r}$ is generated by the $g_{i_{\bullet}, j_{\bullet}}$, and the initial terms of the latter agree with the initial terms of the minors generating $J_{r}$. Now, let $J_{r}^{\prime}$ be the flat degeneration of $J_{r}$ defined as in $\S 15.8$ of [Eis95] by the weight function assigning $\epsilon_{1, j}$ to the variable $x_{i, j}$ if $i \leqslant r_{1}$ and $\epsilon_{2, j}$ to the variable $x_{i, j}$ if $i>r_{1}$. Then, it is clear from the definitions of the $g_{i_{\bullet}, j}$ and of $J_{r}^{\prime}$ that $g_{i_{\bullet}, j_{\bullet}} \in J_{r}^{\prime}$ for each $\left(i_{\bullet}, j_{\bullet}\right)$. But by Theorem 15.17 and Exercise 20.14 of [Eis95], we have that the Hilbert functions of $J_{r}$ and of $J_{r}^{\prime}$ coincide, so we conclude that the Hilbert function of $I_{r}$ is less than or equal to the Hilbert function of $J_{r}$. On the other hand, the universal minors form a Grobner basis for $J_{r}$ (see Theorem 5.3 of [BC03]), and their initial terms agree with those of the $g_{i_{\bullet}, j_{\bullet}}$, so we conclude that in $J_{r} \subseteq$ in $I_{r}$. Again using invariance of Hilbert functions under flat degenerations, we conclude that the Hilbert function of $I_{r}$ is greater than or equal to that of $J_{r}$, so they must be equal, and then we also have in $J_{r}=\operatorname{in} I_{r}$, as desired.

Theorem 1.3 then follows by standard reductions to known results on the initial ideal of universal determinantal ideals. Indeed, we first conclude:

Corollary 2.8. In the case $R=k$ is a field, then $R^{\prime} / I_{r}$ is reduced and CohenMacaulay, with codimension $(d-r)\left(r_{1}+r_{n}-r\right)$ in $R^{\prime}$. If further $s \neq 0$ then $R^{\prime} / I_{r}$ is integral.

Proof. It is well known that $R^{\prime} /$ in $J_{r}$ is reduced and Cohen-Macaulay, of dimension $(d-r)\left(r_{1}+r_{n}-r\right)$; see for instance Theorems 1.10, 5.3 and 6.7 of [BC03]. We conclude the same statements for $R^{\prime} / I_{r}$ by Theorem 2.4, together with Proposition 3.12 of [BC03]. In addition, for $s \neq 0$ we have $R^{\prime} / I_{r} \cong R^{\prime} / J_{r}$, and the latter is integral (again by Theorem 1.10 of [BC03]).

We next find:

Corollary 2.9. In the case that $R=\mathbb{Z}[t]$ and $s=t$, then $R^{\prime} / I_{r}$ is flat over $R$, and integral and Cohen-Macaulay, with codimension $(d-r)\left(r_{1}+r_{n}-r\right)$ in $R^{\prime}$.

Proof. From Theorem 2.4 we have that all fibers of $R^{\prime} / I_{r}$ over $R$ have the same Hilbert function. Since $R$ is reduced, it follows from Exercise 20.14 of [Eis95] that $R^{\prime} / I_{r}$ is flat over $R$. Given flatness, the statements on irreducibility and codimension follows from the corresponding statements on the generic fibers, which is a consequence of the $s \neq 0$ statement of Corollary 2.8. Again using flatness, the statements on Cohen-Macaulyness and reducedness follow from the corresponding statements on fibers (see the Corollaries to Theorems 23.3 and 23.9 of [Mat86]), which is again Corollary 2.8 .

In order to deduce our main theorem from the universal case, we recall Lemma 2.3 of [OT14].

Lemma 2.10. Suppose that $\mathscr{E}_{\bullet}$ is s-linked. Let $c_{i}=\operatorname{rk} f_{i}$ for $i=1, \ldots, n-1$, and by convention set $c_{0}=0, c_{n}=d$. Also, for $j \leqslant i$ set $f_{j, i}=f_{i-1} \circ f_{i-2} \circ \cdots \circ f_{j}$, and for $j \geqslant i$ set $f^{j, i}=f^{i} \circ f^{i+1} \circ \cdots \circ f^{j-1}$. Then locally on $S$, for $i=1, \ldots, n$ there exist subbundles $\mathscr{W}_{i} \subseteq \mathscr{E}_{i}$ of rank $c_{i}-c_{i-1}$ such that:

(i) For $i=2, \ldots, n-1$ we have that

$$
\mathscr{W}_{i} \cap \operatorname{span}\left(\operatorname{ker} f_{i}, \operatorname{ker} f^{i-1}\right)=(0),
$$

and similarly $\mathscr{W}_{1} \cap \operatorname{ker} f_{1}=(0), \mathscr{W}_{n} \cap \operatorname{ker} f^{n-1}=(0)$. 
(ii) For all $j<i$, the restriction of $f_{j, i}$ to $\mathscr{W}_{j}$ is an isomorphism onto a subbundle of $\mathscr{E}_{i}$, and for $j>i$ the restriction of $f^{j, i}$ to $\mathscr{W}_{j}$ is an isomorphism onto a subbundle of $\mathscr{E}_{i}$.

(iii) The natural map

$$
\left(\bigoplus_{j=1}^{i} f_{j, i}\left(\mathscr{W}_{j}\right)\right) \oplus\left(\bigoplus_{j=i+1}^{n} f^{j, i}\left(\mathscr{W}_{j}\right)\right) \rightarrow \mathscr{E}_{i}
$$

is an isomorphism for each $i$.

Proof of Theorem 1.3. The statement is local on $S$, so we may assume that we have $\mathscr{W}_{i} \subseteq \mathscr{E}_{i}$ as in Lemma 2.10, and further that $S=\operatorname{Spec} R$ is affine and the $\mathscr{W}_{i}$ are free. Choose bases of the $\mathscr{W}_{i}$ and use them to induce bases of the $\mathscr{E}_{i}$ via Lemma 2.10 (iii), but with reversed ordering (so that basis elements from $\mathscr{W}_{n}$ come first, and those from $\mathscr{W}_{1}$ last). Choosing arbitrary bases of $\mathscr{F}_{1}$ and $\mathscr{F}_{n}$, we then have that the induced maps

$$
\mathscr{E}_{i} \rightarrow \mathscr{F}_{1} \oplus \mathscr{F}_{n}
$$

are given by matrices of the form of our $A_{\ell}$. These can be viewed as induced by a map $\mathbb{Z}\left[\left\{x_{i, j}\right\}, t\right] \rightarrow R$, and our linked determinantal locus is then the pullback of the universal one under the corresponding morphism $S \rightarrow \operatorname{Spec} \mathbb{Z}\left[\left\{x_{i, j}\right\}, t\right]$. The theorem then follows from Corollary 2.9 by Theorem 3 and Proposition 4 of [EN67] (see also the introduction of [HE71]).

We conclude with a couple of examples showing that the definition of linked determinantal locus is somewhat delicate, in that minor variations will invalidate the conclusion of Theorem 2.4.

Example 2.11. We first observe that a very similar pattern of zeroing out entries in a sequence of matrices can violate the uniqueness of nonzero minors proved in Lemma 2.7. Indeed, in Example 2.3, if we set $\left(A_{3}\right)_{1,3}$ to be $x_{1,3}$ instead of 0 , the minor from the last three columns is $x_{1,3} x_{2,4} x_{3,5}-x_{1,3} x_{3,4} x_{2,5}$, which does not agree with the corresponding minor from $A_{2}$. Moreover, taking the difference yields $x_{2,3} x_{1,4} x_{3,5}-x_{3,3} x_{1,4} x_{2,5}$, and since neither of these monomials is in the initial ideal of $J_{r}$, we see that the conclusion of Theorem 2.4 is also violated in this case.

Example 2.12. We also see that if we zero out monomials from the generators of the standard universal determinantal ideal, even if the initial terms of each generator remain unchanged, in general the initial ideals can change. For instance, if we let $J$ be the ideal generated by the $2 \times 2$ minors of the matrix

$$
A=\left[\begin{array}{lll}
x_{1,1} & x_{1,2} & x_{1,3} \\
x_{2,1} & x_{2,2} & x_{2,3}
\end{array}\right]
$$

and we let

$$
I=\left(x_{1,1} x_{2,2}, x_{1,2} x_{2,3}, x_{1,1} x_{2,3}-x_{2,1} x_{1,3}\right),
$$

then the initial terms of the generators are the same, and since we know the minors are a Grobner basis for $J$, we conclude that

$$
\text { in } I \supseteq \text { in } J=\left(x_{1,1} x_{2,2}, x_{1,2} x_{2,3}, x_{1,1} x_{2,3}\right) .
$$

However, in this case the containment is strict: we see that

$$
x_{2,3}\left(x_{1,1} x_{2,2}\right)-x_{2,2}\left(x_{1,1} x_{2,3}-x_{2,1} x_{1,3}\right)=x_{2,1} x_{2,2} x_{1,3} \notin \text { in } J .
$$




\section{Applichtions to Limit LinEAR SERIES}

We now apply our results to draw conclusions on spaces of limit linear series. Because our results can be applied directly to limit linear series moduli space constructions carried out in [Oss14a], which are explicitly in terms of linked determinantal loci, we have elected to keep the presentation brief and not recall the rather lengthy definitions leading up to the aforementioned constructions. However, we will below briefly recall the Eisenbud-Harris limit linear series definition, so that all objects relevant to our final conclusion, Corollary 3.4, have been defined.

Our main theorem deals with moduli spaces of limit linear series on a oneparameter family $\pi: X \rightarrow B$ of curves somewhat more general than those of compact type. In the general setting, there will be some extra data denoted by $\boldsymbol{n}$ and $\left(\mathscr{O}_{v}\right)_{v}$, and an associated family $\tilde{\pi}: \widetilde{X} \rightarrow \widetilde{B}$, but in the compact type case, these are irrelevant, and in particular we may assume $\tilde{\pi}=\pi$. In either case, we denote the closed point of $\widetilde{B}$ by $b_{0}$, and the special fiber of $\pi$ by $X_{0}$. We will simultaneously treat the case of a single curve, where $\widetilde{B}=b_{0}$.

Theorem 3.1. Suppose that we are in the situation of Theorem 6.1 of [Oss14a] (in particular, with $B$ the spectrum of a DVR), or of Theorem 5.9 of [Oss14a] (in which case $B$ is a point). Suppose also that the space $G_{\bar{w}_{0}}^{r}\left(X_{0}, \boldsymbol{n},\left(\mathscr{O}_{v}\right)_{v}\right)$ of limit linear series on $X_{0}$ has the expected dimension $\rho$ at a given point $z$. Then the limit linear series moduli space $\widetilde{G}_{\bar{w}_{0}}^{\prime r}\left(\tilde{X} / \widetilde{B}, X_{0}, \boldsymbol{n},\left(\mathscr{O}_{v}\right)_{v}\right)$ of Definition 6.3 of [Oss14a] is Cohen-Macaulay at $z$, and flat over $\widetilde{B}$.

In fact, the theorem applies also to higher-dimensional base schemes - see Remark 3.5.

Proof. According to Proposition 6.4 of [Oss14a], $G^{\prime}:=\widetilde{G}_{\bar{w}_{0}}^{\prime r}\left(\widetilde{X} / \widetilde{B}, X_{0}, \boldsymbol{n},\left(\mathscr{O}_{v}\right)_{v}\right)$ is described by the construction in the proof of Theorem 6.1 of [Oss14a]. This construction proceeds by constructing $G^{\prime}$ as a closed subscheme of a scheme $G$ which is smooth over $\widetilde{B}$. Furthermore, $\widetilde{B}$ is regular, so $G$ is likewise regular. The construction is given as an intersection of $(r+1) \operatorname{deg} D$ local equations ensuring vanishing along an auxiliary divisor $D$, together with $|V(\Gamma)-1|$ linked determinantal loci, each of expected codimension $(r+1)(d+\operatorname{deg} D+1-g-(r+1))$. The numbers work out that in order for $G_{\bar{w}_{0}}^{r}\left(X_{0}, \boldsymbol{n},\left(\mathscr{O}_{v}\right)_{v}\right)$ to have dimension $\rho$ at $z$, the aforementioned conditions must intersect with maximal codimension at $z$. It thus follows that each individual condition cuts out a closed subscheme of $G$ of maximal codimension at $z$, and therefore by Theorem 1.3 we conclude that each of these closed subschemes is Cohen-Macaulay at z. By Lemma 4.4 of [HO08] we conclude that the intersection $G^{\prime}$ is likewise Cohen-Macaulay at $z$.

Flatness is nontrivial only in the case that $B$ is positive-dimensional. Then Theorem 6.1 of [Oss14a] implies that $G^{\prime}$ is (universally) open over $B$ at $z$, so every irreducible component containing $z$ dominates $B$. Moreover, Cohen-Macaulayness implies that there are no imbedded components meeting $z$, so since $B$ is the spectrum of a DVR, we conclude that $G^{\prime}$ is flat over $B$ at $z$, as desired.

We now recall the Eisenbud-Harris definition in the compact type case.

Definition 3.2. Given a curve $X_{0}$ of compact type, with dual graph $\Gamma$, for $v \in V(\Gamma)$ let $Z_{v}$ denote the corresponding component of $X_{0}$, and for $e \in E(\Gamma)$, let $P_{e}$ denote the corresponding node. 
Given $r, d>0$, a limit linear series (or more specifically, a limit $\mathfrak{g}_{d}^{r}$ ) on $X_{0}$ consists of a tuple $\left(\mathscr{L}^{v}, V^{v}\right)_{v \in V(\Gamma)}$ of $\mathfrak{g}_{d}^{r} \mathrm{~s}$ on the components $Z_{v}$, satisfying the condition that for each $e \in E(\Gamma)$ connecting vertices $v, v^{\prime}$, we have:

$$
a_{j}^{(e, v)}+a_{r-j}^{\left(e, v^{\prime}\right)} \geqslant d \quad \text { for } j=0, \ldots, r,
$$

where $a^{(e, v)}$ denotes the vanishing sequence of $\left(\mathscr{L}^{v}, V^{v}\right)$ at $P_{e}$.

We say the limit linear series is refined if (3.1) is an equality for all $e \in E(\Gamma)$.

In the compact type case, the Eisenbud-Harris definition of limit linear series leads to a natural scheme structure on the moduli space $G_{d}^{r}\left(X_{0}\right)$ of limit linear seres, as a union of closed subschemes ranging over all possible refined ramification conditions at the nodes. This definition has the advantage of being very amenable to calculations, for instance in verifying reducedness. The alternative definition introduced in $\S 4$ of [Oss14b] and used in the statement of Theorem 3.1 also gives a scheme structure, which in principle could be different. The difficulty in comparing them arises from the union in the Eisenbud-Harris case, as the functor of points of a union cannot be easily described. However, we can now conclude that under typical circumstances, the two scheme structures agree.

Corollary 3.3. If $X_{0}$ is a curve of compact type, suppose that we have the following conditions:

(I) $G_{d}^{r}\left(X_{0}\right)$ has the expected dimension $\rho$;

(II) the refined limit linear series are dense in $G_{d}^{r}\left(X_{0}\right)$;

(III) the Eisenbud-Harris scheme structure on $G_{d}^{r}\left(X_{0}\right)$ is reduced.

Then the Eisenbud-Harris scheme structure on $G_{d}^{r}\left(X_{0}\right)$ coincides with the scheme structure introduced in [Oss14b].

Proof. According to Theorem 3.1, condition (I) implies that the scheme structure of [Oss14b] is Cohen-Macaulay. Thus, in view of condition (III), it is enough to show that the two scheme structures agree on a dense open subset, since CohenMacaulayness will then imply reducedness. But Proposition 4.2.6 of [Oss14b] asserts that the two scheme structures agree on the refined locus, so the desired result follows from condition (II).

For applications such as [CLT] and [CLPT], the key point is the following immediate consequence of Theorem 3.1 and Corollary 3.3.

Corollary 3.4. In the situation of Theorem 6.1 of [Oss14a], suppose further that the special fiber $X_{0}$ is of compact type and the conditions of Corollary 3.3 are satisfied. Then $\widetilde{G}_{\bar{w}_{0}}^{\prime r}\left(\tilde{X} / \widetilde{B}, X_{0}, \boldsymbol{n},\left(\mathscr{O}_{v}\right)_{v}\right)$ is flat and proper over $B$, with special fiber equal to the Eisenbud-Harris scheme structure on $G_{d}^{r}\left(X_{0}\right)$, and generic fiber equal to the classical space $G_{d}^{r}\left(X_{\eta}\right)$ of linear series on $X_{\eta}$.

Remark 3.5. The moduli space construction of [Oss14a] restricted to the case that $B$ was the spectrum of a DVR partly to avoid developing technical hypotheses such as the "almost local" condition of $\S 2.2$ of [Oss14b], and partly because in the non-compact-type case, it is necessary to impose more stringent conditions on the family to ensure that every component of the curve comes from a divisor in the total family. With that said, under suitable hypotheses the construction does generalize to higher-dimensional base schemes $B$, and in this case, Theorem 3.1 also generalizes. The only place where we used that $B$ was the spectrum of a DVR 
was in arguing flatness, but as long as $B$ is regular this argument can be replaced via the use of Theorem 14.2.1 of [GD66] and Proposition 6.1.5 of [GD65].

In the compact type context, it also suffices to assume that our family of curves has a single section (as opposed to a section through each component of the special fiber, as assumed in [Oss14a]). The desired result being etale local, we can then use an etale base change to produce sections through all components of the special fiber.

\section{REFERENCES}

[BC03] Winfried Bruns and Aldo Conca, Grobner bases and determinantal ideals, Commutative algebra, singularities and computer algebra (Siniai 2002), 2003, pp. 9-66.

[CLPT] Melody Chan, Alberto López Martin, Nathan Pflueger, and Montserrat Teixidor i Bigas, Genus of the Brill-Noether curve, in preparation.

[CLT] Abel Castorena, Alberto López Martin, and Montserrat Teixidor i Bigas, Invariants of the Brill-Noether curve, preprint.

[EH86] David Eisenbud and Joe Harris, Limit linear series: Basic theory, Inventiones Mathematicae 85 (1986), no. 2, 337-371.

[EH87a] — Existence, decomposition, and limits of certain Weierstrass points, Inventiones Mathematicae 87 (1987), 495-515.

[EH87b] - The Kodaira dimension of the moduli space of curves of genus $\geqslant 23$, Inventiones Mathematicae 90 (1987), 359-387.

[Eis95] David Eisenbud, Commutative algebra with a view toward algebraic geometry, Graduate Texts in Mathematics, vol. 150, Springer-Verlag, 1995.

[EN67] John Eagon and Douglas Northcott, Generically acyclic complexes and generically perfect ideals, Proceedings of the Royal Society. Series A 299 (1967), 147-172.

[GD65] Alexander Grothendieck and Jean Dieudonné, Éléments de géométrie algébrique: IV. Étude locale des schémas et des morphismes de schémas, seconde partie, Publications mathématiques de l'I.H.É.S., vol. 24, Institut des Hautes Études Scientifiques, 1965.

[GD66] - Éléments de géométrie algébrique: IV. Étude locale des schémas et des morphismes de schémas, troisième partie, Publications mathématiques de l'I.H.É.S., vol. 28, Institut des Hautes Études Scientifiques, 1966.

[HE71] Melvin Hochster and John Eagon, Cohen-Macaulay rings, invariant theory, and the generic perfection of determinantal loci, American Journal of Mathematics 93 (1971), $1020-1058$

[HO08] David Helm and Brian Osserman, Flatness of the linked Grassmannian, Proceedings of the AMS 136 (2008), no. 10, 3383-3390.

[Kho] Deepak Khosla, Moduli spaces of curves with linear series and the slope conjecture, preprint.

[Mat86] Hideyuki Matsumura, Commutative ring theory, Cambridge University Press, 1986.

[Oss14a] Brian Osserman, Limit linear series for curves not of compact type, preprint, 2014.

[Oss14b] — Limit linear series moduli stacks in higher rank, preprint, 2014.

[OT14] Brian Osserman and Montserrat Teixidor i Bigas, Linked alternating forms and linked symplectic Grassmannians, International Mathematics Research Notices 2014 (2014), no. $3,720-744$. 